\title{
The role of primary care in managing chronic kidney disease
}

Chronic kidney disease (CKD) is common, harmful, and treatable. CKD is now recognised as a public health problem because of its association and interactions with other vascular diseases. Primary care has a pivotal role in educating the public, alongside early identification and integrated management of CKD. Times of crisis offer the best environment and opportunities to change what we do for the better. At the very least, the current financial climate demands improved quality of care for the same money. The NHS has traditionally been poor at measuring quality, yet it is essential to know whether what we do adds value or not, and how to maximise implementation of best practice.

Epidemiological studies suggest that up to $10 \%$ of the population have $C K D^{1}$ but some clinicians question the impact and significance of diagnosing a patient with CKD, particularly as the concept of CKD is relatively new and patients with early disease are often asymptomatic. Even individuals with modest reductions in kidney function (estimated glomerular filtration rate $30-44 \mathrm{~mL} / \mathrm{min} / 1.73 \mathrm{~m}^{2}$; CKD Stage 3B), have adjusted hazard ratios for death of 1.8 and are twice as likely to suffer cardiovascular events. ${ }^{2}$ Furthermore, this independent association of CKD with adverse outcomes is graduated, worsening with severity of kidney impairment. Identifying and appropriately managing the 6 million people with CKD in the UK offers real opportunities to improve vascular care, achieve better outcomes, and reduce costs.

To do this we need to see a paradigm shift from secondary care management, focused on rare primary renal diseases in young people and established renal failure, to a chronic disease management model with integration of kidney disease into the management of long-term vascular conditions in primary care. Given the aetiology of CKD, many patients are likely to have a number of significant additional medical and social problems and CKD, viewed as a vascular risk factor, is an easy way of identifying people at risk. The majority of people with CKD will not benefit from secondary care input. CKD management needs primary care specialists, adept at encouraging selfmanagement, emphasising lifestyle and therapeutic priorities in partnership with the patient, taking into account all influential diagnoses and behaviours, using a holistic approach. Management of these patients with multiple comorbidities is challenging, and to measure success requires the establishment of combined quality markers and outcomes to enable effective high quality care. ${ }^{3}$

To facilitate this in the UK, the Quality and Outcomes Framework (QOF) for CKD was introduced in 2006. Currently over $4 \%$ of the adult population have been diagnosed with CKD and are on QOF registers to support better care, creating the first national managed CKD database. This is unparalled worldwide, for which primary care in the UK should be proud.

Despite this success, there is still more we need to achieve, as QOF records demonstrate that we have only identified just under half of the predicted $10 \%$ of the population with CKD. Further, QOF register analysis shows wide variation in documented CKD prevalence between practices and within and across primary care trusts, which is not readily explained by practice characteristics or demographic data. Gallagher et al, via a thorough and interesting qualitative study, describe some clear explanations for the wide variation. ${ }^{4} \quad$ They examined perceptions and knowledge of CKD in primary care and the influence of guidelines and incentives on behaviour in several practices representing the spectrum of QOF ascertainment. NHS Kidney Care and the East Midlands Public Health Observatory recently produced a series of primary care trust profiles, detailing achievements and comparisons in kidney care, demonstrating these aspects, and providing powerful comparator tools. ${ }^{5}$

The concept of CKD and subsequent QOF targets entered primary care without adequate notice, education, and support, which was highlighted by the views recorded by Gallagher and colleagues. This led to a surge of referrals to secondary care, many of which were unnecessary. Ideally, the work undertaken by Gallagher et al's group should have taken place before the addition of CKD to the QOF. Primary care adapted, and the system survived this surge, as the ability and confidence of primary care physicians, nurses, and community pharmacists in CKD management grew.

Once identified, individuals with CKD should receive integrated care to minimise the risk of cardiovascular disease and progression of kidney disease. Through urinalysis, proteinuria (now part of the CKD domain of the QOF), can be detected and albumin:creatinine ratios quantified to identify those individuals with CKD at the highest increased risk of adverse outcomes. Proteinuria, at any level, is a marker of kidney damage and even in the absence of elevated serum creatinine, is an independent marker of increased cardiovascular risk $^{6}$ and indicative of early kidney disease. Thus a simple test in a primary care setting can identify individuals who will benefit from more intensive management, and be used to assess the effectiveness of therapy.

Tight blood pressure control in patients with proteinuria reduces the risk and rate of progression of kidney disease and of cardiovascular events, particularly in older people. Achieving this in everyday practice can be challenging and historically we have not measured methods or mechanisms of improving the quality and effectiveness of care.

Crinson and colleagues examined the existing literature for examples of quality improvement in kidney disease. ${ }^{7}$ Utilising 
particular groups from the Cochrane Collaboration, they scoured the literature for evidence of changes in practice that lead to a clearly defined endpoint: the reduction of systolic blood pressure. They found only nine studies with significant rigour that demonstrated that changes in practice, nurse, or pharmacist can improve blood pressure control, which leads to better outcomes for these patients. In an evidence-based era envisaged by Archie Cochrane of the Cochrane Collaboration, where no-one doubts the utility of randomised controlled trials, we need to accumulate more evidence of innovations relevant to everyday settings in primary care for improvements in outcomes.

The future demands that primary and secondary care work as a seamless service, so that our patients see the right person in the right setting, with care closer to home, patients empowered to manage their condition to the best of their ability, and with support from the appropriate peer or healthcare professional as needed. This vision is outlined clearly in the document Teams without Walls, ${ }^{8}$ and more recently in the Manifesto for Patient Care 2010, produced by the Royal College of General Practitioners. ${ }^{9}$ Collaboration and integration can leverage the best primary care system in the world to provide world class CKD management, not in isolation but as part of holistic care.
The challenges for GPs and primary care teams are to see CKD as part of vascular disease, to take ownership of the problem, and to manage CKD appropriately (in a manner which historically has always proven to be more cost effective than secondary care), with greater impact on prevention by helping to achieve real improvements in outcomes for patients with kidney disease.

\section{Mark Brady,}

Clinical Advisor to the National Clinical Director for Kidney Care, Kidney Care, London.

\section{Donal O'Donoghue,}

National Clinical Director for Kidney Care, Kidney Care, London.

\section{Provenance}

Commissioned; not peer reviewed.

\section{REFERENCES}

1. Stevens PE, O'Donoghue DJ, de Lusignan S, et al. Chronic kidney disease management in the United Kingdom; NEOERICA project results. Kidney Int 2007; 72(1): 92-99.

2. Go AS, Chertow GM, Fan D, et al. Chronic kidney disease and the risks of death, cardiovascular events, and hospitalization. N Engl J Med 2004; 351(13): 1296-1305

3. Levin A, Chaudhry MR, Djurdjev O, et al. Diabetes, kidney disease and cardiovascular disease patients. Assessing care of complex patients using outpatient testing and visits: additional metrics by which to evaluate health care system functioning. Nephrol Dial Transplant 2009; 24(9): 2714-2720.

4. Gallagher H, de Lusignan S, Harris K, Cates C. Qualityimprovement (QI) strategies for the management of hypertension in chronic kidney disease in primary care: a systematic review. Br J Gen Pract 2010; DOI:

10.3399/bjgp10X502164.

5. NHS Kidney Care, East Midlands Public Health Observatory. Kidney disease PCT profiles.

http://www.kidneycare.nhs.uk/OurworkprogrammesKidneyDiseaseinPrimaryCare-

ChronicKidneyDiseaseprofiles-

KidneyDiseasePCTProfiles.cms (accessed 12 May 2010).

6. De Zeeuw D, Parving HH, Henning RH. Microalbuminuria as an early marker for cardiovascular disease. J Am Soc Nephrol 2006; 17(8): 2100-2105.

7. Crinson I, Gallagher H, Thomas N, de Lusignan S. How ready is general practice to improve quality in chronic kidney disease? A diagnostic analysis. $\mathrm{Br} \mathrm{J} \mathrm{Gen}$ Pract 2010; 60: 403-409.

8. Working Party of the Royal College of Physicians, Royal College of General Practitioners, Royal College of Paediatrics and Child Health. Teams without walls: the value of medical innovation and leadership. London: RCP, RCGP \& RCPCH, 2008.

9. Royal College of General Practitioners. Leading the way: high-quality care for all through general practice. A manifesto for patient care 2010. London: RCGP, 2010.

DOI: 10.3399/bjgp10X502065

\section{ADDRESS FOR CORRESPONDENCE}

\section{Mark Brady}

Clinical Advisor to the National Clinical Director for Kidney Care, Kidney Care, Department of Health, 133-155 Wellington House, Waterloo Rd, London, SE1 8UG. Email: markebrady@doctors.org.uk 